Journal of Modern Mathematics and Statistics 13 (2): 28-39, 2019

ISSN: 1994-5388

(C) Medwell Journals, 2019

\title{
Biufrcation Analysis on Infection Disease Treatment by Compartment Models
}

\author{
Tadesse Lamessa \\ Department of Applied Mathematics, College of Natural and Computational Science, \\ Walita Sodo University, Sodo, Ethiopia
}

\begin{abstract}
In this thesis we consider an epidemic model with a constant removal rate of infective individuals is proposed to understand the effect of limited resources for treatment of infective on the disease spread. It is found that it is unnecessary to take such a large treatment capacity that endemic equilibria disappear to eradicate the disease. It is shown that the outcome of disease spread may depend on the position of the initial states for certain range of parameters. It is also shown that the model undergoes a sequence of bifurcations including saddle-node bifurcation, subcritical Hopf bifurcation.
\end{abstract}

Key words: Epidemic model, nonlinear incidence rate, basic reproduction number, local, global stability, bifurcations

\section{INTRODUCTION}

Infectious diseases have ever been a great concern of human kind, since, the very beginning of our history. At present, we still have to deal with plagues and diseases. Mathematical epidemiology contributed to the understanding of the behavior of infectious diseases its impacts and possible future predictions about its spreading. Mathematical models are used in comparing, planning, implementing, evaluating and optimizing various detection, prevention, therapy and control programs.

In some locations, the incidence of some diseases, such as chickenpox, mumps and poliomyelitis, goes up and down every year. Because of the observed periodicity in the incidence of many diseases, there has been great interest in determining how periodic solutions can arise in epidemiological models.

Treatment including isolation or quarantine is an important method to decrease the spread of diseases such as measles, AIDS, tuberculosis and flu (Feng and Thieme, 1995).

By Wu and Feng, Hyman and Li (1998) in classical epidemic models, the removal rate of infective is assumed to be proportional to the number of the infective. This is unsatisfactory because the resources for treatment should be quite large. In fact, every community should have a suitable capacity for treatment. If it is too large, the community pays for unnecessary cost. If it is too small, the community has the risk of the outbreak of the disease. Thus, it is important to determine a suitable capacity for the treatment of a disease.

In this study, we suppose that the capacity for the treatment of a disease in a community is a constant $r$. In order to easily understand its effect we consider a case that the removal rate of infective equals $\mathrm{r}$. This means that we use the maximal treatment capacity to cure or isolate infective, so that, the disease is eradicated. This can occur if the disease is so dangerous that we hope to wipe out it quickly or the disease spreads rapidly, so that, the treatment capacity is insufficient for treatment in a period (flu, for example).

It is also shown that the model undergoes a sequence of bifurcations. The qualitative structure of the flow on the vector field under investigation may change as the parameter values are varied. In particular equilibrium points may be created or destroyed or their stability may change. This qualitative change in the dynamics of the system is called bifurcation and the parameter values at which these changes occur are called bifurcation points. There are different types of bifurcations:

- Saddle node bifurcation

- Pitchfork bifurcation

- Trans critical bifurcations

- Hope bifurcation

In a saddle-node bifurcation as the bifurcation parameter passes through the bifurcation point, two equilibria disappear, so that, there are no equilibria afterward. One of the two equilibria is stable and the other is unstable before they disappear.

In a pitchfork bifurcation, there are two stable equilibria separated by an unstable equilibrium. A system where there two different stable equilibria is said to have a property of bi-stability. When bifurcation point is passed, there is only one stable is equilibrium. This bifurcation is referred to as supercritical pitchfork bifurcation. On the other hand if the stability of supercritical pitchfork bifurcation is reversed, subcritical 
pitchfork bifurcation is obtained where there are two unstable equilibria separated by stable equilibrium, until the bifurcation point is passed.

In trans critical bifurcation, there two equilibria, one stable and the other unstable. When the bifurcation point is passed there is an exchange of stability, the unstable equilibrium becomes stable and the stable equilibrium becomes unstable.

Literature review: Over the past 100 years, mathematics has been used to understand and predict the spread of diseases, relating important public-health questions to basic transmission parameters. From prehistory to the present day, diseases have been a source of fear and superstition. A comprehensive picture of disease dynamics requires a variety of mathematical tools from model creation to solving differential equations to statistical analysis. Although, mathematics has been so far, done quite well in dealing with epidemiology but there is no denying that there are certain factors which still lack proper mathematization (Brauer and van den Driessche, 2001).

Almost all mathematical models of diseases start from the same basic premise, that the population can be subdivided into a set of distinct classes, dependent upon their experience with respect to the disease. One line of investigation classifies individuals as one of Susceptible (S); those who have never had the illness and can catch it, Infectious (I): those who currently have the illness and are contagious; Recovered (R): individuals who have acquired a permanent immunity to the disease. Such a model is termed a SIR a model. Epidemic models have been studied by many researchers. The first model which computes the theoretical number of individuals infected with a contagious illness in a closed population over time was proposed by Kermack and McKendrick (1927), Xiao and Ruan (2007), Derrick and Van Den Driessche (1993).

SIR Model is easily written using Ordinary Differential Equations (ODEs) which implies a deterministic model (no randomness is involved, the same starting conditions give the same output) with continuous time (as opposed to discrete time). The rate of new infections can thus be defined as ${ }_{\text {}}$ SI where ${ }_{\text {B }}$ is a parameter for infectivity. Infected individuals are assumed to recover with a constant probability at any time which translates into a constant per capita recovery rate that, we denote with $r$ and thus, an overall rate of recovery rI. Based on these assumptions, we can draw the scheme of the model (Fig. 1).

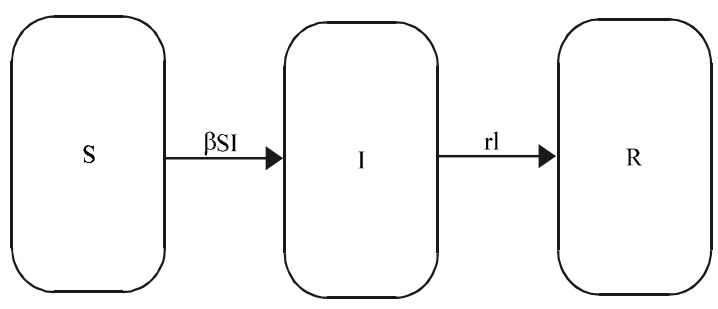

Fig. 1: The transformation chart of SIR Model

The scheme translation to ordinary differential equation:

$$
\begin{aligned}
& \frac{\mathrm{ds}}{\mathrm{dt}}=-\beta S I \\
& \frac{\mathrm{dI}}{\mathrm{dt}}=\beta S I-\mathrm{rI} \\
& \frac{\mathrm{dR}}{\mathrm{dt}}=\mathrm{rI}
\end{aligned}
$$

where, $S(t), R(t), I(t)$ represent the number of susceptible, infective and recover individuals at time, respectively. The parameters and $r$ are called transmission rate and recovery rate, respectively. The interpretation of this model is straight forward. The population of susceptible (healthy) individuals diminishes through their interaction with the infective ones, the number of which correspondingly increases through the mechanism. On the other hand, the population of infective individuals diminishes, since, some individuals are cured and thus, populate the class of recovered. The model is derived under three main assumptions:

A closed population (no births, no deaths and no migration)

. Spatial homogeneity

Disease is transmission by contact between susceptible and infected individuals. The size of the population $(\mathrm{S}+\mathrm{I}+\mathrm{R})$ is therefore, constant and equal to the initial population size which we denote with the parameter $\mathrm{N}$.

AfterKermack-McKendrick Model, different epidemic models have been proposed and studied in the literature (Capasso and Serio, 1978; Ruan and Wang, 2003; Liu a a., 1987; Hethcote as., 1981; Hethcote and Tudor, 1980; Hethcote and Levin, 1989, Hethcote and Van den Driessche, 1991; Beretta and Takeuchi, 1995, 1997; Beretta

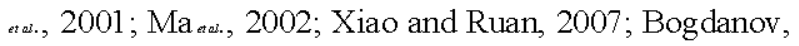
$1981 \mathrm{a}, \mathrm{b})$.

\section{MODEL FORMULATION AND ANALYSIS}

In this study, we present model description, formulation and analysis. We consider that the total 
population is constant and that a small number of infected individuals are introduced into a large population.

Compartment models: The models of the behavior of an infectious disease in a large population of people consider each individual as being in a particular state. These states are often called compartments and the corresponding models are called compartment models. A compartmental model is one for which the individuals in a population are classified into compartments depending on their status with regard to the infection under study.

A compartment model provides a framework for the study of transport between different compartments of a system. The simplest compartment models assume a person can be in one of only three states, either Susceptible (S), S, the susceptible which can get the disease or I; the Infected who have the disease and can transmit it or $\mathrm{R}$, the removed, either recovered and immune or isolated. The three state model is called the SIR Model.

Let $\mathrm{S}(\mathrm{t}), \mathrm{I}(\mathrm{t}), \mathrm{R}(\mathrm{t})$ be represent the number of susceptible, infective and recovered individuals at time, respectively. If we think about the process of a disease that it's the SIR framework, we have a flow of individuals from the susceptible group to the infected group and then to the removed group.

The assumptions and parameter: The following assumptions and parameter are made in order to construct the model:

The population has constant size $\mathrm{N}$, the natural death rate of the population is $\mathrm{d}$, the natural recovery rate of the infective individuals $r, A$ is the recruitment rate of the population, the transmission rate denoted ${ }_{B}$.

The number of infected increases at a rate proportional to both the number of infected and the susceptible; ${ }_{\beta}$ SI with $>0$. The number of the susceptible decreases at this same rate

The rate of removal of infected to the $R$ class is proportional to the number of infected only; ${ }_{\text {I }}$ with $>0$. The number of removed increases at this same rate.

The incubation time is negligible, so that, a susceptible that catches the disease becomes infectious immediately.

If we assume that individuals are mixed randomly then each potential transmission may be from an infected person to a susceptible person which results in a new infected person. Or a transmission may occur from an infected person to another infected person which results is nothing happening, since, the person is already infected. Or the potential transmission may occur from an infected person to a recovered or immune person. In this case again nothing changes.
The model equation: Now by using ordinal differential equation and above assumption; The mathematical model of SIR Model is:

$$
\begin{aligned}
& \frac{\mathrm{ds}}{\mathrm{dt}}=\mathrm{A}-\mathrm{dS}-\beta \mathrm{SI}, \\
& \frac{\mathrm{dI}}{\mathrm{dt}}=\beta \mathrm{SI}-(\mathrm{d}+\gamma) \mathrm{I}-\mathrm{h}(\mathrm{I}), \\
& \frac{\mathrm{dR}}{\mathrm{dt}}=\gamma \mathrm{I}+\mathrm{h}(\mathrm{I})-\mathrm{dR}
\end{aligned}
$$

where, all parameter are positive and $h(\mathrm{I})$ is the removal rate of infective individuals due to the treatment of infective. We suppose that the treated infective become recovered when they are treated in treatment sites. Suppose that:

$$
h(I)=\left\{\begin{array}{l}
r \text { for } I>0 \\
0 \text { for } I=0
\end{array}\right.
$$

where, $r>0$ is a constant and it represents the capacity of treatment for infective. This means that we use a constant removal rate for the infective until the disease disappears.

Suppose that $(\mathrm{S}(\mathrm{t}), \mathrm{I}(\mathrm{t}), \mathrm{R}(\mathrm{t}))$ is a solution of $(1)$. And $N(t)$ is the population at any given time. This must always be positive because a negative population does not biologically meaning. Therefore, $\mathrm{N}>0$, this implies $\mathrm{S}(\mathrm{t})>0$, $I(t)>0, R(t)>0$ for $0_{s} t<t$. Thus, the lower bound for $S, I$ and $\mathrm{R}$ is 0 . To find the upper bound of the system we consider the system Eq. 1 in the total population size is:

$$
\begin{aligned}
& \mathrm{N}=\mathrm{S}+\mathrm{I}+\mathrm{R} \text { and } \mathrm{N}^{\prime}=(\mathrm{S}+\mathrm{I}+\mathrm{R})^{\prime}= \\
& \mathrm{A}-\mathrm{dS}-\mathrm{dI}-\mathrm{dR}=\mathrm{A}-\mathrm{d}(\mathrm{S}+\mathrm{I}+\mathrm{R}) \leq \mathrm{A}-\mathrm{dN}
\end{aligned}
$$

The solution of the linear differential equation then becomes:

$$
N(t)=N(0) e^{-d t}+\frac{A}{d}\left(1-e^{-d t}\right)
$$

It follows that $\lim _{t \rightarrow \infty} N(t) \leq \frac{A}{d}$ that means $N$ is bounded. Let:

$$
\Gamma=\left\{\begin{array}{l}
(S, I, R) \in R^{3}: S+I+R \leq \frac{A}{d} \\
\text { such that } S \geq 0, I \geq 0, R \geq 0
\end{array}\right\}
$$

Therefore, $\mathrm{r}$ is region positively invariant with respect to system (Eq. 1). The purpose of this study is to show that this removal rate has significant effects on the dynamics of. Since, the first 2 equations in Eq. 1 are independent of the variable $\mathrm{R}$. It suffices to consider the following reduced model: 


$$
\begin{aligned}
& \frac{\mathrm{ds}}{\mathrm{dt}}=\mathrm{A}-\mathrm{dS}-\beta \mathrm{SI}, \\
& \frac{\mathrm{dI}}{\mathrm{dt}}=\beta \mathrm{SI}-(\mathrm{d}+\gamma) \mathrm{I}-\mathrm{r}
\end{aligned}
$$

It is assumed that all the parameters are positive constants.

Equlibriua and their stability: Now we investigate the existence of equilibria of system (Eq. 3). System (Eq. 3) has always a disease-free equilibrium and unique endemic equilibrium exist. The equilibrium points of the system can be obtained by equating the rate of changes to zero:

$$
\begin{aligned}
& \frac{\mathrm{ds}}{\mathrm{dt}}=\frac{\mathrm{dI}}{\mathrm{dt}}=\frac{\mathrm{dR}}{\mathrm{dt}}=0, \text { this mean; } \\
& \left\{\begin{array}{l}
\mathrm{A}-\mathrm{dS}-\beta \mathrm{SI}=0 \\
\beta \mathrm{SI}-(\mathrm{d}+\gamma) \mathrm{I}-\mathrm{r}=0
\end{array}\right.
\end{aligned}
$$

The disease free equilibrium: The equilibrium state in the absence of infection is known as disease free equilibrium. Such that, $\mathrm{I}=0$ in the first equation of system (Eq. 4) then, we get $S=A / d$. Therefore, the disease free equilibrium is:

$$
\mathrm{E}_{0}\left(\frac{\mathrm{A}}{\mathrm{d}}, 0\right)
$$

The basic reproduction number (R): The basic reproduction number of an infectious disease is a one of the fundamental concepts in mathematical biology. It is defined as the average number of secondary infections produced when one infected individual is introduced into a host population where everyone is susceptible.

The stability analysis of disease-free equilibrium determines the thresholds (reproductive number) of the epidemic. By linearizd the system about the point (E.). The Jacobean matrix of the system is given by:

$$
\left.J\right|_{\left(\frac{A}{d}, 0\right)}=\left[\begin{array}{cc}
-d-\lambda & \beta \frac{A}{d} \\
0 & \beta \frac{A}{d}-d-\gamma-\lambda
\end{array}\right]
$$

The Eigen values are:

$$
\lambda_{1,2}=-\mathrm{d},-\left(\beta \frac{\mathrm{A}}{\mathrm{d}}-\mathrm{d}-\gamma\right)
$$

Thus, the basic reproduction number $\mathrm{R}$ is often as the threshold quantity that determines whether or not an infectious disease will spread through a population. Since, $\mathrm{S}=\mathrm{A} / \mathrm{d}$ for $\mathrm{I}=0, \mathrm{R}$ is the product of the number $\mathrm{A} / \mathrm{d}$ of susceptible at the disease-free equilibrium state, the transmission coefficient and the average residence time $1 / \mathrm{d}+_{r}$ in the infectious individuals class. The basic reproductive number of the system (Eq. 3 ) is:

$$
\mathrm{R}_{0}=\frac{\beta \mathrm{A}}{\mathrm{d}(\mathrm{d}+\gamma)}
$$

If $\mathrm{R}<1$ there is a disease free equilibrium which is asymptotically stable and the infections dies-out. If $R>1$ the usual situation is there is an endemic equilibrium which is asymptotically stable and the infections persist.

The endemic equilibrium state: The equilibrium state with the presence of infection (i.e., I.0) is known as endemic equilibrium or non-zero equilibrium. Now to find the endemic equilibrium we substitute: $\mathrm{S}=\mathrm{A} / \mathrm{d}+_{\mathrm{B}} \mathrm{I}$ into the second equation of system to obtain the quadratic Eq. 5:

$$
-\beta(d+\gamma) I^{2}+\left(\beta A-r \beta-\gamma d-d^{2}\right) I-r d=0
$$

Since, the basic reproductive number of the system Eq. 3 is:

$$
\mathrm{R}_{0}=\frac{\beta \mathrm{A}}{\mathrm{d}(\mathrm{d}+\gamma)}, \text { Let } \quad \mathrm{H}=\frac{\beta \mathrm{r}}{\mathrm{d}(\mathrm{d}+\gamma)}
$$

Then, Eq. 5 can be write as:

$$
\frac{\beta}{d} I^{2}-\left(R_{0}-1-H\right) I+\frac{r}{d+\gamma}=0
$$

$\mathrm{R}$. is the reproduction number of Eq. 3 in the absence of the removal rate:

$$
\text { Case } 1 \text { If } \mathrm{R}_{0} \leq 1 \text { or } 0<\sqrt{\mathrm{R}_{0}}-1<\sqrt{\mathrm{H}}
$$

Then, Eq. 6 does not have a positive solution:

$$
\text { Case } \left.2 \text { If } \mathrm{R}_{0}>1 \text { and } \sqrt{\mathrm{R}_{0}}-1\right)^{2}=\mathrm{H}
$$

Then, Eq. 6 has 1 unique positive solution:

$$
\text { Case } 3 \text { If } \mathrm{R}_{0}>1 \text { and }\left(\sqrt{\mathrm{R}_{0}}-1\right)^{2}>\mathrm{H}>0
$$

Then, Eq. 6 has two positive solutions. Thus, Eq. 3 does not have a positive equilibrium if R.1. Furthermore, Eq. 8 implies that Eq. 3 has one positive endemic equilibrium and Eq. 9 implies that Eq. 3 has two endemic equilibria. If $\mathrm{N}=\mathrm{S}+1$, we have:

$$
\frac{\mathrm{dN}_{1}}{\mathrm{dt}}=\mathrm{A}-\mathrm{r}-\gamma \mathrm{\gamma}-\mathrm{dN} \mathrm{N}_{1} \leq \mathrm{A}-\mathrm{r}-\mathrm{dN} \mathrm{N}_{1}
$$


Again that positive solution of Eq. 3 is bounded. Note that the non negative I-axis repels positive solutions of Eq. 3 and that there is no equilibrium on the non negative S-axis. If R.1 or Eq. 7 holds it follows that I (t) becomes 0 in finite time, i.e., the disease disappears in a finite time. Now, we propose the following assumption:

$$
\mathrm{H}_{1}=\mathrm{R}_{0}>1 \text { and } 0<\mathrm{H}<\left(\sqrt{\mathrm{R}_{0}}-1\right)^{2}
$$

If $\mathrm{H}$ then, Eq. 3 two endemic equilibrium, $\mathrm{E}=(\mathrm{S}, \mathrm{I})$ and $\mathrm{E},=\left(\mathrm{S}, \mathrm{I}_{\mathrm{r}}\right)$, where:

$$
\begin{aligned}
& I_{1}=\frac{d}{2 \beta}\left(R_{0}-1-H-\sqrt{\left(R_{0}-1-H\right)^{2}-4 H}, S_{1}=\frac{A}{d+\beta I_{1}}\right. \\
& I_{2}=\frac{d}{2 \beta}\left(R_{0}-1-H+\sqrt{\left(R_{0}-1-H\right)^{2}-4 H}, S_{2}=\frac{A}{d+\beta I_{2}}\right.
\end{aligned}
$$

Although, the endemic equilibria occur under the assumption $(\mathrm{H})$, we will show that the disease can disappear in a range of the parameters. This means that it is unnecessary to increase the removal rate $\mathrm{r}$ to $\mathrm{H}>\left(\sqrt{\mathrm{R}_{0}}-1\right)^{2}$ to make the disease disappear.

\section{STABILITY BY LINEARIZATION}

For most dynamical systems the equilibrium point (fixed point) of a system of non-linear differential equations plays an important role in the analysis of the models we examine the behavior of the solutions of the autonomous near an isolated critical point $(x, y$.) where $f$ $(\mathrm{x}, \mathrm{y})=.\mathrm{g}(\mathrm{x}, \mathrm{y})=$.0 .

$$
\begin{aligned}
& \frac{d x}{d t}=f(x, y) \\
& \frac{d y}{d t}=g(x, y)
\end{aligned}
$$

The first step in investigating the behaviour of tra jectories of the system (Eq. 10) near a critical point ( $\mathrm{x}, \mathrm{y}$.) is by approximating the non-linear system with an appropriate linear system, whose trajectories are easy to investigate. The crucial question is whether the tra jectories of the linearized system are good approximations to those of the nonlinear system. First, we explain what is meant by a nonlinear system being close to a linear system. We assume that the functions $\mathrm{f}$ and $\mathrm{g}$ are continuously differentiable in a neigh-bourhood of $(x, y)$. Then, the Taylor formula for $\mathrm{f}$ and $\mathrm{g}$ about the critical point ( $\mathrm{x}, \mathrm{y}$ ) gives:

$$
\begin{aligned}
& \frac{d x}{d t}=f_{x}\left(x_{0}, y_{0}\right)\left(x-x_{0}\right)+f_{y}\left(x_{0}, y_{0}\right)\left(y-y_{0}\right)+F\left(x-x_{0}, y-y_{0}\right) \\
& \frac{d y}{d t}=g_{x}\left(x_{0}, y_{0}\right)\left(x-x_{0}\right)+g_{y}\left(x_{0}, y_{0}\right)\left(y-y_{0}\right)+G\left(x-x_{0}, y-y_{0}\right) \\
& u=x-x_{0} v=y-y_{0} \text {, sothat, } \frac{d x}{d t}=\frac{d u}{d t} \text { and } \frac{d y}{d t}=\frac{d v}{d t}
\end{aligned}
$$

Then in matrix form, Eq. 10a becomes:

$$
\left[\begin{array}{c}
\mathrm{u}^{\prime} \\
\mathrm{v}^{\prime}
\end{array}\right]=\left[\begin{array}{ll}
\mathrm{f}_{\mathrm{z}}\left(\mathrm{x}_{0}, \mathrm{y}_{0}\right) & \mathrm{f}_{\mathrm{y}}\left(\mathrm{x}_{0}, \mathrm{y}_{0}\right) \\
\mathrm{g}_{\mathrm{x}}\left(\mathrm{x}_{0}, \mathrm{y}_{0}\right) & \mathrm{g}_{\mathrm{y}}\left(\mathrm{x}_{0}, \mathrm{y}_{0}\right)
\end{array}\right]\left[\begin{array}{l}
\mathrm{u} \\
\mathrm{v}
\end{array}\right]+\left[\begin{array}{c}
\mathrm{F}(\mathrm{u}, \mathrm{v}) \\
\mathrm{G}(\mathrm{u}, \mathrm{v})
\end{array}\right]
$$

The matrix is the jacobean matrix of the system in Eq. 10 evaluated at critical point $(\mathrm{x}, \mathrm{y}$ ) :

$$
J=\left[\begin{array}{ll}
f_{x}\left(x_{0}, y_{0}\right) & f_{y}\left(x_{0}, y_{0}\right) \\
g_{x}\left(x_{0}, y_{0}\right) & g_{y}\left(x_{0}, y_{0}\right)
\end{array}\right]
$$

Definition: The autonomous system (Eq. 10) is called almost linear at the point provided that it can be put in the form:

$$
\begin{aligned}
& \frac{d x}{d t}=a\left(x-x_{0}\right)+b\left(y-y_{0}\right)+F\left(x-x_{0}, y-y_{0}\right) \\
& \frac{d y}{d t}=c\left(x-x_{0}\right)+d\left(y-y_{0}\right)+G\left(x-x_{0}, y-y_{0}\right)
\end{aligned}
$$

Where:

$$
\begin{aligned}
& \mathrm{a}=\mathrm{f}(\mathrm{x}, \mathrm{y} .) \\
& \mathrm{b}=\mathrm{f}(\mathrm{x}, \mathrm{y} .) \\
& \mathrm{c}=\mathrm{g}(\mathrm{x}, \mathrm{y} .) \\
& \mathrm{d}=\mathrm{g}(\mathrm{x}, \mathrm{y} .) \\
& \mathrm{F}=(\mathrm{x}-\mathrm{x}, \mathrm{y}-\mathrm{y} .) \\
& \mathrm{G}=(\mathrm{x}-\mathrm{x}, \mathrm{y}-\mathrm{y} .)
\end{aligned}
$$

Have the property that:

$$
\lim _{\left\|(x, y)-\left(x_{0}, y_{0}\right)\right\| \rightarrow 0} \frac{\left\|F\left(x-x_{0}, y-y_{0}\right)\right\|}{\left\|(x, y)-\left(x_{0}, y_{0}\right)\right\|}=0
$$

That is in the neigh-boarhound of ( $x, y$.) the expressions $F(x-x, y-y$.$) and G(x-x, y-y$.$) are small in$ comparison with $\|(\mathrm{x}, \mathrm{y})-(\mathrm{x}, \mathrm{y})\|$ which is itself small. Thus, when $(x, y)$ is near the point of $(x, y)$ the nonlinear system in Eq. 10 is "close" to the linearized system. The system in (x., y.) is called the linearization of about the critical point:

$$
\frac{d x}{d t}=a x+b y, \frac{d y}{d t}=c x+d y
$$

Theorem 3.5:1: $\mathrm{E}$ ( $\mathrm{S}, \mathrm{I}$ ) is saddle whenever it exists and $\mathrm{E},\left(\mathrm{S}_{,}, \mathrm{I}\right)$ is center. 
Proof: We begin by analyzing the stability of these two equilibria. The Jacobin matrix of Eq. 3:

$$
J=\left[\begin{array}{ll}
\frac{\partial S^{\prime}}{\partial S} & \frac{\partial S^{\prime}}{\partial \mathrm{I}} \\
\frac{\partial \mathrm{I}^{\prime}}{\partial \mathrm{S}} & \frac{\partial \mathrm{I}^{\prime}}{\partial \mathrm{I}}
\end{array}\right]
$$

Now, at (S., I.), we get:

$$
J_{1}=\left[\begin{array}{cc}
-d-\beta I_{1} & -\beta S_{1} \\
\beta I_{1} & \beta S_{1}-d-\gamma
\end{array}\right]
$$

Note that, from Eq. $3 \mathrm{~A}-\mathrm{dS} \mathrm{S}_{1}=\beta \mathrm{S}_{1} \mathrm{I}_{1}=(\mathrm{d}+\gamma) \mathrm{I}_{1}+\mathrm{r}$, we have $\mathrm{S}_{1}=\frac{\mathrm{A}-(\mathrm{d}+\gamma) \mathrm{I}_{1}-\mathrm{r}}{\mathrm{d}}$, thus, we have det:

$$
\begin{aligned}
& \left(\mathrm{J}_{1}\right)=\left(-\mathrm{d}-\beta \mathrm{I}_{1}\right)(\beta \mathrm{S} 1-\mathrm{d}-\gamma)+\beta^{2} \mathrm{~S}_{1} \mathrm{I}_{1}= \\
& -\beta \mathrm{A}+2 \beta(\mathrm{d}+\gamma) \mathrm{I}_{1}+\mathrm{r} \beta+\mathrm{d}(\mathrm{d}+\gamma)= \\
& \mathrm{d}(\mathrm{d}+\gamma)\left[-\mathrm{R}_{0}+\frac{2 \beta \mathrm{I}_{1}}{\mathrm{~d}}+\mathrm{H}+1\right]= \\
& \mathrm{d}(\mathrm{d}+\gamma)\left[-\mathrm{R}_{0}+\frac{2 \beta}{\mathrm{d}}\left(\frac{\mathrm{d}}{2 \beta}\left(\mathrm{R}_{0}-1-\mathrm{H}-\sqrt{\left(\mathrm{R}_{0}-1-\mathrm{H}\right)^{2}-4 \mathrm{H}}+\mathrm{H}+1\right)\right]=\right. \\
& \mathrm{d}(\mathrm{d}+\gamma)\left[-\mathrm{R}_{0}+\mathrm{R}_{0}-\sqrt{\left(\mathrm{R}_{0}-1-\mathrm{H}\right)^{2}-4 \mathrm{H}}\right]- \\
& \mathrm{d}(\mathrm{d}+\gamma)\left[\sqrt{\left(\mathrm{R}_{0}-1-\mathrm{H}\right)^{2}-4 \mathrm{H}}\right]<0
\end{aligned}
$$

It follows that (S, I.) is saddle point. And the Jacobin matrix of Eq. 13 at $\left(S_{,}, I_{1}\right)$ is:

$$
J \mid\left(S_{2}, I_{2}\right)=\left[\begin{array}{cc}
-d-\beta I_{2} & -\beta S_{2} \\
\beta I_{2} & \beta S_{2}-(d+\gamma)
\end{array}\right]
$$

By the same argument, we obtain det:

$$
\left(\mathrm{J}_{2}\right)=\mathrm{d}(\mathrm{d}+\gamma)\left[\sqrt{\left(\mathrm{R}_{0}-1-\mathrm{H}\right)^{2}-4 \mathrm{H}}\right]>0
$$

Thus, E, (S, I, ) it's center.

Theorem 3.2.2: If $H$ hold. Then, Eq. 4:

$$
E_{2} \text { Stable if either } \beta A-3 d^{2}-d \gamma-\frac{2 d^{3}}{\gamma} \leq \beta r
$$

Or:

$$
\begin{aligned}
& \beta \mathrm{r}<\beta \mathrm{A}-3 \mathrm{~d}^{2}-\mathrm{d} \gamma-\frac{2 \mathrm{~d}^{3}}{\gamma} \\
& \beta \mathrm{r}<\frac{1}{2}\left[2 \beta \mathrm{A}+(2 \mathrm{~d}+\gamma)(\mathrm{d}+\gamma)\left(1-\sqrt{1+\frac{4 \beta \mathrm{A}}{(d+\gamma)^{2}}}\right)\right]
\end{aligned}
$$

$\mathrm{E}_{2}$ unstable if :

$$
\begin{aligned}
& \beta \mathrm{r}<\beta \mathrm{A}-3 \mathrm{~d}^{2}-\mathrm{d} \gamma-\frac{2 \mathrm{~d}^{3}}{\gamma} \text { and } \\
& \beta \mathrm{r}>\frac{1}{2}\left[2 \beta \mathrm{A}+(2 \mathrm{~d}+\gamma)(\mathrm{d}+\gamma)\left(1-\sqrt{1+\frac{4 \beta \mathrm{A}}{(\mathrm{d}+\gamma)^{2}}}\right)\right]
\end{aligned}
$$

Proof: Since, we have $S_{2}=\frac{A-(d+\gamma) I_{2}-r}{d}$ and the trace of $J$, is:

$$
\begin{aligned}
& \operatorname{tr}\left(\mathrm{J}_{2}\right)=-2 \mathrm{~d}-\beta \mathrm{I}_{2}+\beta \mathrm{S}_{2}-\gamma= \\
& -2 \mathrm{~d}-\beta \mathrm{I}_{2}+\beta\left(\frac{\mathrm{A}-(\mathrm{d}+\gamma) \mathrm{I}_{2}-\mathrm{r}}{\mathrm{d}}\right)-\gamma= \\
& \frac{-2 \mathrm{~d}^{2}-\mathrm{d} \beta \mathrm{I}_{2}+\beta \mathrm{A}-\beta(\mathrm{d}+\gamma) \mathrm{I}_{2}-\beta \mathrm{r}-\mathrm{d} \gamma}{\mathrm{d}}= \\
& -\left(\frac{2 \mathrm{~d} \beta+\gamma \beta}{\mathrm{d}}\right) \mathrm{I}_{2}-\frac{2 \mathrm{~d}^{2}-\beta \mathrm{A}+\beta \mathrm{r}+\gamma \mathrm{d}}{\mathrm{d}}
\end{aligned}
$$

Thus, the trace is negative if $2 \mathrm{~d}^{-}{ }_{\mathrm{B}} \mathrm{A}+{ }_{\mathrm{B}} \mathrm{r}+{ }_{\mathrm{\gamma}} \mathrm{d}, 0$ let:

$$
2 d^{2}-\beta A+\beta r+\gamma d<0
$$

Let us find the condition under which the $\operatorname{tr}\left(\mathrm{J}_{0}\right)=0$ set:

$$
D_{1} \triangleq-\frac{d(d+\gamma)}{\beta(2 d+\gamma)}\left(\frac{d}{d+\gamma}+1-R_{0}+H\right)
$$

Equation 14 implies that $\operatorname{tr}(\mathrm{J})=0$ is equivalent to:

$$
\begin{aligned}
& I_{2}=-\frac{2 d^{2}-\beta A+\beta r+\gamma d}{\beta(2 d+\gamma)}=D_{1} \\
& \text { If } D_{2} \triangleq-\frac{2 d}{2 d+\gamma}+\frac{\gamma}{2 d+\gamma}\left(R_{0}-1-H\right)
\end{aligned}
$$

It follows from the definition of $\mathrm{I}$, that $\operatorname{tr}\left(\mathrm{J}_{\mathrm{J}}\right)=0$ is equivalent to:

$$
\mathrm{D}_{2}=\sqrt{\left(\mathrm{R}_{0}-1-\mathrm{H}\right)^{2}-4 \mathrm{H}}
$$

Thus, the set of $\operatorname{tr}(\mathrm{J})=$,0 is empty if:

$$
\mathrm{H} \geq \mathrm{R}_{0}-1-\frac{2 \mathrm{~d}}{\gamma}
$$

Let:

$$
\mathrm{H}<\mathrm{R}_{0}-1-\frac{2 \mathrm{~d}}{\gamma}
$$

Taking squares on both sides of Eq. 17 and simplifying the resulting equation, we obtain: 


$$
D_{3} \underline{\underline{\Delta}} \mathrm{r}^{2} \beta+\left(-3 \gamma d-2 \beta A-\gamma^{2}-2 d^{2}\right) r+\beta A^{2}-2 A d^{2}=0
$$

The using quadratic equation:

$$
\begin{aligned}
& \mathrm{r}=\frac{1}{2 \beta}[(2 \beta \mathrm{A}+(2 \mathrm{~d}+\gamma)(\mathrm{d}+\gamma) \pm \\
& \left.\sqrt{(2 \beta \mathrm{A}+(2 \mathrm{~d}+\gamma)(\mathrm{d}+\gamma))^{2}-4 \beta \mathrm{A}\left(\beta \mathrm{A}^{2}-2 \mathrm{Ad}^{2}\right)}\right] \\
& \mathrm{r}=\frac{1}{2 \beta}\left[2 \beta \mathrm{A}+(2 \mathrm{~d}+\gamma)(\mathrm{d}+\gamma) \pm(2 \mathrm{~d}+\gamma)(\mathrm{d}+\gamma) \sqrt{\frac{(\mathrm{d}+\gamma)^{2}+4 \beta \mathrm{A}}{(\mathrm{d}+\gamma)^{2}}}\right.
\end{aligned}
$$

Hence:

$$
r=\frac{1}{2 \beta}\left[2 \beta \mathrm{A}+(2 \mathrm{~d}+\gamma)(\mathrm{d}+\gamma)\left(1 \pm \sqrt{1+\frac{4 \beta \mathrm{A}}{(\mathrm{d}+\gamma)^{2}}}\right)\right]
$$

In view (Eq. 15):

$$
\mathrm{r}=\frac{1}{2 \beta}\left[2 \beta \mathrm{A}+(2 \mathrm{~d}+\gamma)(\mathrm{d}+\gamma)\left(1-\sqrt{1+\frac{4 \beta \mathrm{A}}{(\mathrm{d}+\gamma)^{2}}}\right)\right]
$$

By definition of D, we D. have:

$$
\begin{aligned}
& \operatorname{tr}\left(\mathrm{J}_{2}\right)=-\left(\frac{2 \mathrm{~d} \beta+\gamma \beta}{\mathrm{d}}\right) \mathrm{I}_{2}-\mathrm{D}_{1}= \\
& -\left(\frac{2 \mathrm{~d}+\gamma}{2}\right)\left(\sqrt{\left(\mathrm{R}_{0}-1-\mathrm{H}\right)^{2}-4 \mathrm{H}}-\mathrm{D}_{2}\right)
\end{aligned}
$$

Thus, Eq. 18 implies tha $\operatorname{tr}(\mathrm{J})<0$. Therefore, E, is stable if 21 holds. Since:

$$
\left.\left(\mathrm{R}_{0}-1-\mathrm{H}\right)^{2}-4 \mathrm{H}\right)-\mathrm{D}_{2}^{2}=\frac{4 \beta}{\mathrm{d}(2 \mathrm{~d}+\gamma)(\mathrm{d}+\gamma)} \mathrm{D}_{3}
$$

It follows that $\operatorname{tr}\left(\mathrm{J}_{\mathrm{J}}\right)<0$, if Eq. 12 is valid and that $\operatorname{tr}$ $(\mathrm{J})>$,0 , if Eq. 13 hold.

Local stability of the disease-free and endemic steady states: This study explains the method of local (or linear) stability analysis of equilibria in models with several differential equations. We obtain in this section is a generalization of the stability.

If system does not have a limit cycle it is easy to classify its dynamical behaviour. If is unstable, any positive semi-orbit except the two equilibria and the stable manifolds of intersects the positive S-axis in finite time. A typical phase portrait is shown in Fig. 4.

If $\mathrm{E}$, is stable, there is a region whose boundary includes the two stable manifolds of $\mathrm{E}$. such that any positive semi-orbit inside this region tends to $\mathrm{E}$, as $\mathrm{t}$ tends to infinity and any positive semi-orbit outside this region meets the positive $\mathrm{S}$-axis in a finite time. A typical phase portrait is shown in Fig. 2-4.

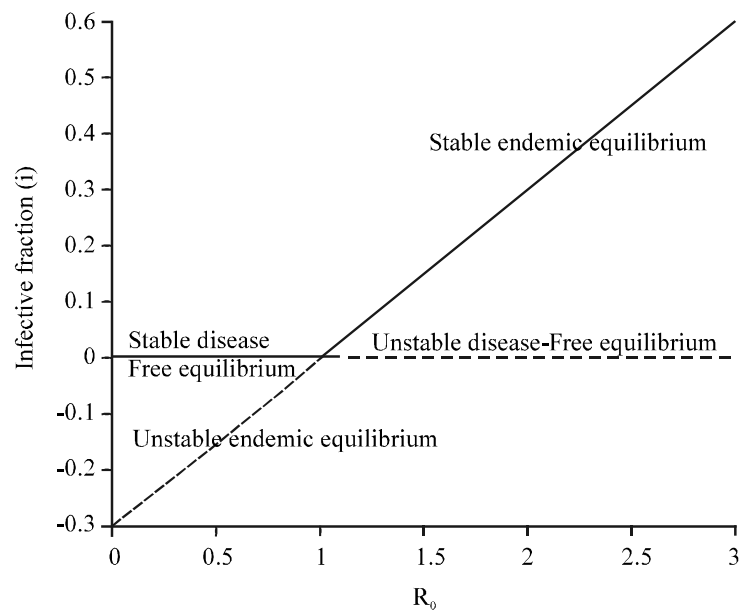

Fig. 2: A schematic of a bifurcation for SIR Model at there is a trans-critical bifurcation

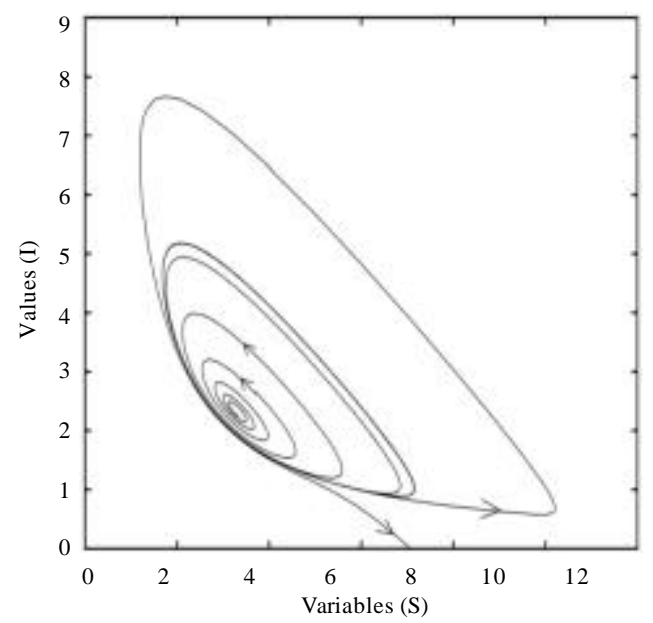

Fig. 3: An unstable periodic solution exist where $\mathrm{A}=8$, $\mathrm{d}=0.1, \mathrm{\beta}=1, \mathrm{r}=1, \mathrm{r}=5.1$

In the absence of the infectious diseases the model has unique diseases-free state $\mathrm{E}$. To establish the local stability of meet at $\mathrm{E}(\mathrm{A} / \mathrm{d}, 0)$, we use Jacobean of the model evaluate at $\mathrm{E}$ then determine the Eigen values of the corresponding Jacobean which are a function of the model parameters. The Jacobean matrix of the system Eq. 1 is given by:

$$
\left.J\right|_{\left(\frac{A}{d}, 0\right)}=\left[\begin{array}{cc}
-d-\lambda & \beta \frac{A}{d} \\
0 & \beta \frac{A}{d}-d-\gamma-\lambda
\end{array}\right]
$$

The Eigen values are:

$$
\lambda_{1,2}=-d,-\left(\beta \frac{A}{d}-d-\gamma\right)
$$




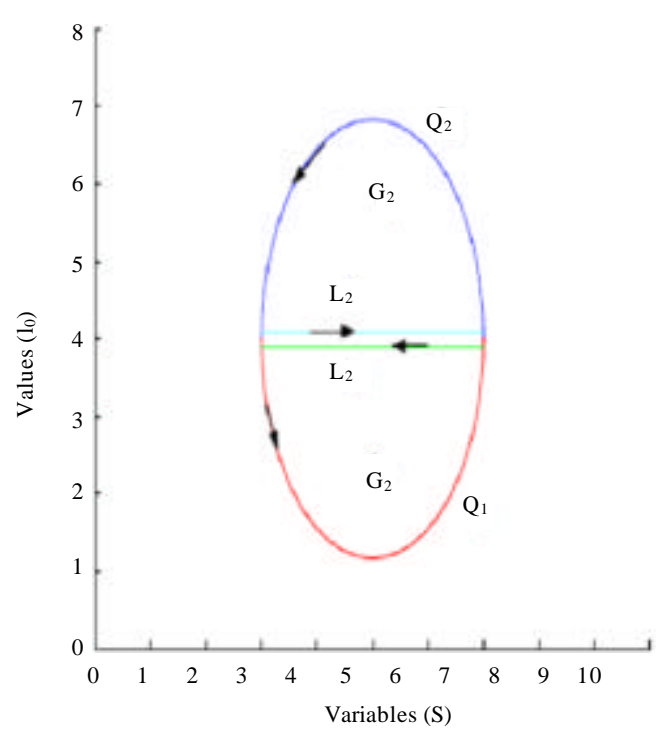

Fig. 4: The diagram of $Q$. and Q. split from the limit cycle and the diagram of $\mathrm{G}$. and $\mathrm{G}$.

In the following theorem established the local stability of the disease free steady state.

Theorem 3.5.1.1: The disease-free equilibrium $\mathrm{E}$, is locally asymptotically stable if $\mathrm{R}<1$ and an unstable if $\mathrm{R}>1$.

Proof: Since, from above ${ }_{2}<0$ the diseases free steady state $E$ is locally asymptotically stable if ${ }{ }_{2}<0$. Nothing that ${ }_{2}<0$ if and only if $\mathrm{R}<1$. If $\mathrm{R}>1$ then ${ }_{2}$, is positive. Therefore, E. is unstable.

Theorem 3.5.1.2: The endemic equilibrium E. (S., I.) is locally asymptotically stable.

Proof: We begin by analyzing the stability of these two equilibria. The Jacobin matrix of Eq. 3:

$$
J=\left[\begin{array}{ll}
\frac{\partial S^{\prime}}{\partial \mathrm{S}} & \frac{\partial \mathrm{S}^{\prime}}{\partial \mathrm{I}} \\
\frac{\partial \mathrm{I}^{\prime}}{\partial \mathrm{S}} & \frac{\partial \mathrm{I}^{\prime}}{\partial \mathrm{I}}
\end{array}\right]
$$

Now by inserting (S., I.), we get:

$$
J_{2}=\left[\begin{array}{cc}
-d-\beta I_{2} & -\beta S_{2} \\
\beta I_{2} & \beta S_{2}-d-\gamma
\end{array}\right]
$$
Note that fromEq. $3 A-d S=S \mathrm{~S}=\left(\mathrm{d}+{ }_{v}\right) \mathrm{H}$, we wave $\mathrm{S}_{2}=\frac{\mathrm{A}-(\mathrm{d}+\gamma) \mathrm{I}_{2}-\mathrm{r}}{\mathrm{d}}$.
Then trace of J, is:

$$
\begin{aligned}
& \operatorname{tr}\left(\mathrm{J}_{2}\right)=-2 \mathrm{~d}-\beta \mathrm{I}_{2}+\beta \mathrm{S}_{2}-\gamma= \\
& -2 \mathrm{~d}-\beta \mathrm{I}_{2}+\beta\left(\frac{\mathrm{A}-(\mathrm{d}+\gamma) \mathrm{I}_{2}-\mathrm{r}}{\mathrm{d}}\right)-\gamma= \\
& \frac{-2 \mathrm{~d}^{2}-\mathrm{d} \beta \mathrm{I}_{2}+\beta \mathrm{A}-\beta(\mathrm{d}+\gamma) \mathrm{I}_{2}-\beta \mathrm{r}-\mathrm{d} \gamma}{\mathrm{d}}= \\
& -\left(\frac{2 \mathrm{~d} \beta+\gamma \beta}{\mathrm{d}}\right) \mathrm{I}_{2}-\frac{2 \mathrm{~d}^{2}-\beta \mathrm{A}+\beta \mathrm{r}+\gamma \mathrm{d}}{\mathrm{d}}
\end{aligned}
$$

Thus, the trace is negative if $2 \mathrm{~d}_{\rightarrow} \mathrm{A}+{ }_{\mathrm{r}} \mathrm{r}+{ }_{v} \mathrm{~d} .0$ and the det:

$$
\begin{aligned}
& \left(\mathrm{J}_{2}\right)=\left(-\mathrm{d}-\beta \mathrm{I}_{2}\right)\left(\beta \mathrm{S}_{2}-\mathrm{d}-\gamma\right)+\beta^{2} \mathrm{~S}_{2} \mathrm{I}_{2}= \\
& -\beta \mathrm{A}+2 \beta(\mathrm{d}+\gamma) \mathrm{I}_{2}+\mathrm{r} \beta+\mathrm{d}(\mathrm{d}+\gamma)= \\
& \mathrm{d}(\mathrm{d}+\gamma)\left[-\mathrm{R}_{0}+\frac{2 \beta \mathrm{I}_{2}}{\mathrm{~d}}+\mathrm{H}+\mathrm{l}\right]= \\
& \mathrm{d}(\mathrm{d}+\gamma)\left[-\mathrm{R}_{0}+\frac{2 \beta}{\mathrm{d}}\left(\frac{\mathrm{d}}{2 \beta}\left(\mathrm{R}_{0}-1-\mathrm{H}+\sqrt{\left(\mathrm{R}_{0}-1-\mathrm{H}\right)^{2}-4 \mathrm{H}}+\mathrm{H}+1\right)\right]=\right. \\
& \mathrm{d}(\mathrm{d}+\gamma)\left[-\mathrm{R}_{0}+\mathrm{R}_{0} \sqrt{\left(\mathrm{R}_{0}-\mathrm{l}-\mathrm{H}\right)^{2}-4 \mathrm{H}}\right]= \\
& \mathrm{d}(\mathrm{d}+\gamma)\left[\sqrt{\left(\mathrm{R}_{0}-1-\mathrm{H}\right)^{2}-4 \mathrm{H}}\right]>0 \\
& \operatorname{det}\left(\mathrm{J}_{2}\right)=\mathrm{d}(\mathrm{d}+\gamma)\left[\sqrt{\left(\mathrm{R}_{0}-1-\mathrm{H}\right)^{2}-4 \mathrm{H}}\right]>0
\end{aligned}
$$

$$
\operatorname{det}\left(\mathrm{J}_{2}\right)=\mathrm{d}(\mathrm{d}+\gamma)\left[\sqrt{\left(\mathrm{R}_{0}-1-\mathrm{H}\right)^{2}-4 \mathrm{H}}\right]>0
$$

Hence, the trace $(\mathrm{J})<$.0 and $\operatorname{det}(\mathrm{J})>$,0 . This implies that the egien value of Jacobean matrix $\mathrm{J}(\mathrm{E}$ ) have negative real part. This means E. is locally asymptotically stable.

Global stability of the disease-free and endemic steady state: The global stability of both the disease-free and endemic steady state is proven by Lyapunov functions.

Theorem 3.5.2.1: If, $R_{1} 1$, then the diseases-free steady state $\mathrm{E} .(\mathrm{A} / \mathrm{d}, 0)$ is globally asymptotically stable and if $\mathrm{R}>1$ then $\mathrm{E}$, is unstable. Let proof by Lyapunov functions let:

$$
\begin{aligned}
& \mathrm{v}(\mathrm{S}, \mathrm{I})=\mathrm{I} \\
& \frac{\mathrm{dv}}{\mathrm{dt}}=\mathrm{I} \frac{\mathrm{ds}}{\mathrm{dt}}+\frac{\mathrm{dI}}{\mathrm{dt}} \\
& \beta \mathrm{A}-\mathrm{d}(\mathrm{d}+\gamma) \mathrm{I}-\mathrm{rd} \\
& (\mathrm{d}+\gamma)\left[\mathrm{R}_{0} \mathrm{~S}-1\right] \mathrm{I}-\mathrm{rd} \leq 0, \text { for } \mathrm{R}_{0} \leq 1 \\
& \text { If } \mathrm{R}_{0}<1, \frac{\mathrm{dv}}{\mathrm{dt}}=0 \text {, iff } \mathrm{I}=0 \\
& \text { If } \mathrm{R}_{0}=1, \frac{\mathrm{dv}}{\mathrm{dt}}=0 \text {, iff } \mathrm{S}=\frac{\mathrm{A}}{\mathrm{d}}
\end{aligned}
$$


Hence, $\mathrm{v}$ is a Lyapunov function in $\mathrm{r}$. Therefore, E. is globally asymptotically stable.

Theorem 3.5.2.2: If the endemic equilibrium of system (Eq. 1) exists and if is $\mathrm{H}$. holds, then $\mathrm{E}=(\mathrm{S}, \mathrm{I}$ ) it is globally asymptotically stable. Let proof by Lyapunov functions:

$$
\begin{aligned}
& \text { Let } \mathrm{V} ; \Gamma \rightarrow \mathrm{R}: \Gamma=\{(\mathrm{S}, \mathrm{I})\} \in \Gamma \mid \mathrm{S}>0, \mathrm{I}>0 \text { and } \\
& \mathrm{S}-\mathrm{S}_{2}>0, \mathrm{I}_{2}>0 \text {, for } \mathrm{h}(\mathrm{I})=0 \\
& \text { Let } \mathrm{V}(\mathrm{S}, \mathrm{I})=\mathrm{w}_{1}\left(\mathrm{~S}-\mathrm{S}_{2} \ln \left(\frac{\mathrm{S}}{\mathrm{S}_{2}}\right)+\right. \\
& \mathrm{w}_{2}\left(\mathrm{I}-\mathrm{I} \mathrm{I}_{2} \ln \left(\frac{\mathrm{I}}{\mathrm{I}_{2}}\right) \text { for some } \mathrm{w}_{1}, \mathrm{w}_{2}>0\right. \\
& \frac{\mathrm{dv}}{\mathrm{dt}}=\mathrm{w}_{1}\left(\mathrm{~S}-\mathrm{S}_{2}\right) \frac{\mathrm{ds}}{\mathrm{dt}}+\mathrm{w}_{2}\left(\mathrm{I}-\mathrm{I}_{2}\right) \frac{\mathrm{dI}}{\mathrm{dt}} \\
& \frac{\mathrm{dv}}{\mathrm{dt}}=\mathrm{w}_{1}\left(\mathrm{~S}-\mathrm{S}_{2}\right)\left(-\beta \mathrm{I}-\mathrm{d}+\frac{\mathrm{A}}{\mathrm{S}}\right)+\mathrm{w}_{2}\left(\mathrm{I}-\mathrm{I}_{2}\right)(\beta \mathrm{S}-(\mathrm{d}+\gamma)) \\
& \frac{\mathrm{dv}}{\mathrm{dt}}=\mathrm{w}_{1}\left(\mathrm{~S}-\mathrm{S}_{2}\right)(\mathrm{A}-\beta \mathrm{SI}-\mathrm{dS})+\mathrm{w}_{2}\left(\mathrm{I}-\mathrm{I}_{2}\right)(\beta \mathrm{SI}-(\mathrm{d}+\gamma) \mathrm{I})
\end{aligned}
$$

From the equation at equilibrium:

$$
\begin{aligned}
& -d=\beta I_{2}-\frac{A}{S_{2}},-(d+\gamma)=-\beta S_{2} \\
& \frac{\mathrm{dv}}{\mathrm{dt}}=\mathrm{w}_{1}\left(\mathrm{~S}-\mathrm{S}_{2}\right)\left(-\beta \mathrm{I}+\beta \mathrm{I}_{2}+\frac{\mathrm{A}}{\mathrm{S}}-\frac{\mathrm{A}}{\mathrm{S}_{2}}\right)+\mathrm{w}_{2}\left(\mathrm{I}-\mathrm{I}_{2}\right)\left(\beta \mathrm{S}-\beta \mathrm{S}_{2}\right) \\
& \frac{\mathrm{dv}}{\mathrm{dt}}=\beta\left[\mathrm{w}_{1}\left(\mathrm{~S}-\mathrm{S}_{2}\right)\left(\mathrm{I}_{2}-\mathrm{I}\right)+\mathrm{A}\left(\frac{\mathrm{S}_{2}-\mathrm{S}}{\mathrm{SS}_{2}}\right)+\mathrm{w}_{2}\left(\mathrm{I}-\mathrm{I}_{2}\right)\left(\mathrm{S}-\mathrm{S}_{2}\right)\right] \\
& \frac{\mathrm{dv}}{\mathrm{dt}}=\beta\left[\mathrm{w}_{1}\left(\mathrm{~S}-\mathrm{S}_{2}\right)\left(\mathrm{I}_{2}-\mathrm{I}\right)+\mathrm{A}\left(\frac{\mathrm{S}-\mathrm{S}_{2}}{\mathrm{SS}_{2}}\right)+\mathrm{w}_{2}\left(\mathrm{I}-\mathrm{I}_{2}\right)\left(\mathrm{S}-\mathrm{S}_{2}\right)\right] \\
& \frac{\mathrm{dv}}{\mathrm{dt}}=-\beta\left[\mathrm{w}_{1}\left(\mathrm{~S}-\mathrm{S}_{2}\right)\left(\mathrm{I}-\mathrm{I}_{2}\right)-\mathrm{w}_{1} \mathrm{~A}\left(\frac{\mathrm{S}_{2}-\mathrm{S}}{\mathrm{SS}_{2}}\right)\right]+\beta \mathrm{w}_{2}\left(\mathrm{I}-\mathrm{I}_{2}\right)\left(\mathrm{S}-\mathrm{S}_{2}\right) \\
& \frac{\mathrm{dv}}{\mathrm{dt}}=\beta\left[\mathrm{w}_{2}-\mathrm{w}_{1}\left(\mathrm{~S}-\mathrm{S}_{2}\right)\left(\mathrm{I}-\mathrm{I}_{2}\right)-\mathrm{w}_{1} \mathrm{~A} \frac{\left(\mathrm{S}-\mathrm{S}_{2}\right)^{2}}{\mathrm{SS}_{2}} \text { for } \mathrm{w}_{1}=\mathrm{w}_{2}=1\right. \\
& \frac{\mathrm{dv}}{\mathrm{dt}}=-\mathrm{A} \frac{\left(\mathrm{S}-\mathrm{S}_{2}\right)^{2}}{\mathrm{SS}_{2}} \leq 0 \\
& \frac{\mathrm{dv}}{\mathrm{dt}}=0 \text { iff } \mathrm{S}=\mathrm{S}_{2}
\end{aligned}
$$

Hence, its global stable. Therefore, the endemic equilibrium state $\mathrm{E}$ is globally asymptotical stable.

Bifurcation analysis: The main purpose of this study is to get an insight into how the dynamics of the system changes depending on the system parameter.

If parameter is allowed to vary, the dynamics of the differential system may change. An equilibrium can become unstable and a period solution may appear or a new stable equilibrium may appear making the previous equilibrium unstable.

The change in the qualitative character of a solution as a control parameter is varied is known as a bifurcation and the parameter values at which they occur are called bifurcation points. The main purpose is to get an insight into how the dynamics of the system changes depending on the system parameter.

Note that the endemic equilibrium point is only positive (and in the domain of interest) if ${ }_{B} \mathrm{~A}>\mathrm{d}\left(\mathrm{d}++_{\mathrm{v}}\right)$. Linear stability analysis shows that diseases-free steady state is locally asymptotically stable if ${ }_{\mathrm{B}} \mathrm{A}<\mathrm{d}\left(\mathrm{d}++_{\mathrm{v}}\right)$ and an unstable if ${ }_{B} A>d\left(d+_{v}\right)$ while the endemic stead state is locally asymptotically stable ${ }_{\mathrm{B}} \mathrm{A}>\mathrm{d}\left(\mathrm{d}+{ }_{\mathrm{v}}\right)$ and an unstable ${ }_{\beta} \mathrm{A}<\mathrm{d}\left(\mathrm{d}++_{\mathrm{v}}\right)$.

Furthermore, at ${ }_{n} \mathrm{~A}=\mathrm{d}\left(\mathrm{d}+_{\mathrm{v}}\right)$, so, there is a transcritical bifurcation. If we draw the stability region of SIR Model for the disease free equilibrium and endemic equilibrium.

If $\mathrm{R}>1$ each individual produces, on average, less than one new infected individual and hence, the disease dies out. And if $R>1$, each individual produces more than one new infected individual and hence, the disease is able to invade the susceptible population. There is the threshold condition $\mathrm{R}$ = 1 is equivalent to the threshold condition describes at ${ }_{\beta} \mathrm{A}=\mathrm{d}\left(\mathrm{d}+{ }_{\mathrm{v}}\right)$. So, describes $\mathrm{R}=1 \mathrm{a}$ trans-critical bifurcation as shown Fig. 2 .

Where the disease-free equilibrium and endemic equilibrium point collide and exchange stability. It is also clear that a unique stable endemic equilibrium arises from the bifurcation point $\mathrm{R}$, and increases as $\mathrm{R}$ increases ( $\mathrm{B}$ increases by fixing $\mathrm{A}$ and $\mathrm{d}$ ), thus, it shown that infectious free steady state exists for all $\mathrm{R}$ while an endemic infection only exist for $\mathrm{R}>1$. Let us verity that existence of a Hopf bifurcation in Eq. 3 and determine its direction:

$$
\text { Set } A_{0}=\frac{1}{2 \beta}\left[2 \beta A+(2 d+\gamma)(d+\gamma)\left(1-\sqrt{1+\frac{4 \beta A}{(d+\gamma)^{2}}}\right)\right]
$$

Theorem 3.6: If, $H$ is exist. Assume further that:

$$
\mathrm{r}<\frac{\beta \mathrm{A}-3 \mathrm{~d}^{2}-\mathrm{d} \gamma-\frac{2 \mathrm{~d}^{3}}{\gamma}}{\beta}
$$

Then there is a family of unstable limit cycles if $r$ is less than and close to A. i.e., a subcritical Hopf bifurcation occurs when $r$ passes through the critical value $\mathrm{A}$. 
Proof: Let $\mathrm{r}=\mathrm{A}$. Then the $\operatorname{tr}(\mathrm{J})=0$. It follows from Eq. 16 that:

$$
\begin{aligned}
& \operatorname{tr}\left(\mathrm{J}_{2}\right)=-2 \mathrm{~d}-\beta \mathrm{I}_{2}+\beta \mathrm{S}_{2}-\gamma=0 \\
& =-2 \mathrm{~d}-\beta \mathrm{I}_{2}+\beta\left(\frac{\mathrm{A}}{\mathrm{d}+\beta \mathrm{I}_{2}}\right)-\gamma \\
& \beta^{2} \mathrm{I}_{2}^{2}-(3 \mathrm{~d} \beta+\gamma \beta) \mathrm{I}_{2}+2 \mathrm{~d}^{2}-\beta \mathrm{A}+\gamma \mathrm{d}=0 \\
& \beta \mathrm{I}_{2}^{2}-(3 \mathrm{~d}+\gamma) \mathrm{I}_{2}+-\mathrm{A}+\frac{\gamma d+2 \mathrm{~d}^{2}}{\beta}=0 \\
& \mathrm{I}_{2}=\frac{1}{2 \beta}\left[-(3 \mathrm{~d}+\gamma)+\sqrt{(3 \mathrm{~d}+\gamma)^{2}-4\left(2 \mathrm{~d}^{2}-\beta \mathrm{A}+\gamma \mathrm{d}\right)}\right] \\
& \mathrm{I}_{2}=\frac{\mathrm{d}+\gamma}{\beta}\left(-\frac{\mathrm{d}}{\mathrm{d}+\gamma}-\frac{1}{2}+\frac{1}{2} \sqrt{1+\frac{4 \beta \mathrm{A}}{(d+\gamma)^{2}}}\right. \\
& \mathrm{S}_{2}=\frac{\mathrm{d}+\gamma}{2 \beta}\left(1+\sqrt{1+\frac{4 \beta \mathrm{A}}{(\mathrm{d}+\gamma)^{2}}}\right)
\end{aligned}
$$$$
\text { From above trance }\left(\mathrm{J}_{2}\right)=0 \text {. And } \operatorname{det}\left(\mathrm{J}_{2}\right)>0
$$

Now by using above information, we get:

$$
\begin{aligned}
& \lambda_{1,2}= \pm \mathrm{i} \sqrt{\mathrm{d}(\mathrm{d}+\gamma) \sqrt{\left(\mathrm{R}_{0}-1-\mathrm{H}\right)^{2}-4 \mathrm{H}},} \\
& \text { Now let } \omega=\sqrt{\operatorname{det}\left(\mathrm{J}_{2}\right)} \omega=\sqrt{\mathrm{d}(\mathrm{d}+\gamma) \sqrt{\left(\mathrm{R}_{0}-1-\mathrm{H}\right)^{2}-4 \mathrm{H}}}
\end{aligned}
$$

Then the eigen values of $\left(\mathrm{J}_{2}\right)$ are $x_{1 .}= \pm \pm_{1} \mathrm{i}$, perform coordinate transformation by $\mathrm{x}=\mathrm{S}-\mathrm{S}$, $\mathrm{y}=\mathrm{I}-\mathrm{I}$, then system Eq. 3 becomes:

$$
\left\{\begin{array}{l}
\frac{d x}{d t}=-\left(d+\beta I_{2}\right) x-\beta S_{2} y-\beta x y \\
\frac{d y}{d t}=\beta I_{2} x+\beta S_{2}-(d+\varepsilon) y+\beta x y
\end{array}\right.
$$

Setting $\mathrm{x}={ }_{-\beta} \mathrm{S}, \mathrm{V}, \mathrm{y}={ }_{ } \mathrm{U}+\left(\mathrm{d}+{ }_{\mathrm{\beta}}\right) \mathrm{V}$ and using tr $(\mathrm{J})=-2 \mathrm{~d}-$

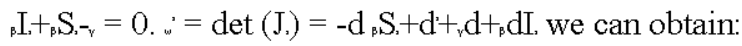

$$
\left\{\begin{array}{l}
\frac{d U}{d t}=-\omega V+F(U, V), \\
\frac{d V}{d t}=\omega U+G(U, V)
\end{array}\right.
$$

Where:

$$
\begin{aligned}
& \mathrm{F}(\mathrm{U}, \mathrm{V})=\frac{\beta \mathrm{V}\left(-\beta \mathrm{S}_{2}+\beta \mathrm{I}_{2}+\mathrm{d}\right)\left(\omega \mathrm{U}+\mathrm{dV}+\beta \mathrm{I}_{2} \mathrm{~V}\right)}{\omega} \\
& \mathrm{G}(\mathrm{U}, \mathrm{V})=-\beta \mathrm{V}\left(\omega \mathrm{U}+\mathrm{dV}+\beta \mathrm{I}_{2} \mathrm{~V}\right)
\end{aligned}
$$

We know that, $\operatorname{tr}(\mathrm{J})=0$, so, we get $\mathrm{G}(\mathrm{U}, \mathrm{V})=\frac{\omega \mathrm{F}(\mathrm{U}, \mathrm{V})}{\mathrm{d}+\gamma}$ :

$$
\begin{aligned}
& \mu=\frac{1}{16}\left[F_{U U U}+F_{U V V}+G_{U U V}\right]+\frac{1}{16 \omega} \\
& {\left[F_{U V}\left(F_{U U}+F_{V V}\right)-G_{U V}\left(G_{U U}+G_{V V}\right)-F_{U U} G_{U U}+F_{V V} G_{V V}\right]}
\end{aligned}
$$

By some calculations, we obtain:

$$
\mu=-\frac{\left.\omega^{2}-2 d \beta I_{2}-2 d \gamma I_{2}\right)}{8(d+\gamma)^{2}}
$$

Note that, ${ }_{\beta} \mathrm{S},{ }_{\beta} \mathrm{I}+\mathrm{d}=-\mathrm{d}-{ }_{\gamma}$. We have:

$$
\mu=\frac{\beta^{2}\left(d+\beta I_{2}\right)\left(2 d^{2}+3 \gamma d+\gamma^{2}+2 \beta S_{2}+\gamma \beta I_{2}\right)}{8 \omega^{2}}>0
$$

Then the conclusion of this theorem follows from $\mathrm{L}$. Perko As an example, we fix $\mathrm{A}=8, \mathrm{~d}=0.1{ }_{, \beta}=1,{ }_{\gamma}=1$. Then, we obtain $\left(\sqrt{\mathrm{R}_{0}}-1\right) \mathrm{d}\left(\mathrm{d}+_{\mathrm{v}}\right)=6.2338,{ }_{\beta} \mathrm{A}-3 \mathrm{~d}-\mathrm{d}_{\mathrm{r}}-2 \mathrm{~d} / \mathrm{v}_{\mathrm{v}}=$ 7.868 and $\mathrm{A} .=5.2023$.

We know that there is an unstable limit cycle when is less than and nearfrom theorem 4.6 which is shown that there is an unstable limit cycle when decrease from 5.2023 which (Fig. 3).

It is easy to verify that positive solutions of are ultimately bounded. Note that the nonnegative S-axis is positively invariant and that the nonnegative I-axis repels positive solutions of Eq. 1. Since, E is asymptotically stable it follows from the Bendixson theorem that every positive solution of approaches as $t$ approaches infinity. The limit cycles of play crucial roles on the structure of dynamical behaviors of the model. For example, if there is no limit cycle and its endemic equilibrium is unique, the unique endemic equilibrium is globally stable. For this reason we adopt Dulac functions to obtain conditions for the non-existence of a limit cycle in Eq. 1. Let denote the right-hand sides of Eq. 1 by $f$ and $f$.

Lemma 3.7: If there is a continuous function $D$ in $R$; such that $\mathrm{D}$ is continuously differentiate when I.I. and

$$
\frac{\partial\left(\mathrm{Df}_{1}\right)}{\partial \mathrm{S}}+\frac{\partial\left(\mathrm{Df}_{2}\right)}{\partial \mathrm{I}}<0 \text { when } \mathrm{I} \neq \mathrm{I}_{0}
$$

Then system is does not have a limit cycle.

Proof: Without loss of generality we suppose that $Q$ is a limit cycle of Eq. 1 across the line $\mathrm{I}=\mathrm{I}$, with a period $\mathrm{T}$. We denote its part below $\mathrm{I}=\mathrm{I}$. by $\mathrm{Q}$. and its part above $\mathrm{I}=\mathrm{I}$. by

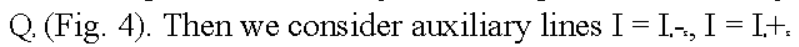
denoted by L, L, respectively where $>0$ is sufficiently small. Let $\mathrm{G}$. denote the region enclosed by $\mathrm{Q}$. and $\mathrm{L}$. and let $\mathrm{G}$. denote the region enclosed by $\mathrm{Q}$, and L. If ${ }_{n}$, and ${ }_{n}$, be the bound a $G$. and $G$, respectively with the directions indicated in Fig. 4 then Green's theorem implies that: 


$$
\begin{gathered}
\int_{G_{1}} \int\left[\frac{\partial\left(\mathrm{Df}_{1}\right)}{\partial \mathrm{S}}+\frac{\partial\left(\mathrm{Df}_{2}\right)}{\partial \mathrm{I}}\right] \mathrm{dsdI} \oint_{\eta_{2}} f \mathrm{f}\left(\mathrm{f}_{1} \mathrm{dI}-\mathrm{f}_{2} \mathrm{ds}\right), \\
\int_{\mathrm{G}_{2}} \int\left[\frac{\partial\left(\mathrm{Df} \mathrm{f}_{1}\right)}{\partial \mathrm{S}}+\frac{\partial\left(\mathrm{Df}_{2}\right)}{\partial \mathrm{I}}\right] \mathrm{dsdI}=\int_{\eta_{2}} \mathrm{D}\left(\mathrm{f}_{1} \mathrm{dI}-\mathrm{f}_{2} \mathrm{ds}\right)
\end{gathered}
$$

If $\mathrm{G}_{\mathrm{e}} \mathrm{G} 1$, we have:

$$
\delta:=\int_{G_{2}} \int\left[\frac{\partial\left(\mathrm{Df}_{1}\right)}{\partial \mathrm{S}}+\frac{\partial\left(\mathrm{Df}_{2}\right)}{\partial \mathrm{I}}\right] \mathrm{dsdI}<0
$$

It follows from Eq. 25 that:

$$
\delta>\int_{\eta_{1}} D\left(f_{1} d I-f_{2} d s\right)+\int_{\eta_{2}} D\left(f_{1} d I-f_{2} d s\right)
$$

Taking $=0$ in Eq. 26, we obtain:

$$
0>\delta>\int_{Q} D\left(f_{1} d I-f_{2} d s\right)=\int_{0}^{T} D\left(f_{1} f_{2}-f_{2} f_{1} d s\right)=0
$$

This implies non-existence of the limit cycle Q. Now, we apply lemma 4.7 to obtain sufficient conditions for the nonexistence of a limit cycle in Eq. 1 .

Theorem 3.8: System does not have a limit cycle if $\mathrm{r}<\mathrm{d}$.

Proof: We know $r$ is a positive invariant set, so, we have $\mathrm{S}<\mathrm{A} / \mathrm{d}$. Take a dulac function $\mathrm{D}=1 / \mathrm{SI}$. Then, we have:

$$
\begin{aligned}
& \frac{\partial\left(\mathrm{Df}_{1}\right)}{\partial \mathrm{S}}+\frac{\partial\left(\mathrm{Df}_{2}\right)}{\partial \mathrm{I}}=-\frac{\mathrm{A}}{\mathrm{S}^{2} \mathrm{I}}+\frac{\mathrm{r}}{\mathrm{SI}^{2}} \\
& =\frac{1}{\mathrm{~S}^{2} \mathrm{I}^{2}}(-\mathrm{AI}+\mathrm{Sr}), \text { from }(1) \text { for } \mathrm{I}>0
\end{aligned}
$$

We have:

$$
\begin{aligned}
& \leq \frac{1}{\mathrm{~S}^{2} \mathrm{I}^{2}}\left(-\mathrm{AI}+\frac{\mathrm{rA}}{\mathrm{d}}\right) \\
& \leq \frac{\mathrm{A}}{\mathrm{dS}^{2} \mathrm{I}^{2}}(\mathrm{r}-\mathrm{d})<0, \text { since, } \mathrm{r}<\mathrm{d}
\end{aligned}
$$

Hence, system Eq. 1 does not have a limit cycle.

Theorem 4.8: Implies that there is no limit cycle in system Eq. 1, if $\mathrm{r}<\mathrm{d}$ is to say if the treatment for infective is less than the death rate, there will no limit cycle. We now present a different condition for non-existence of the limit cycle of Eq. 1 is independent of the treatment.

Since, $\mathrm{E}$, is unstable and there is no limit cycle, any orbit except the two endemic equilibria and the stable manifolds of $\mathrm{E}$ meets the positive $\mathrm{S}$-axis in finite time, i.e., the disease becomes extinct in finite time. If the assumption (i) of theorem 4.2 .2 holds, since, there is no limit cycle in Eq. 1 and $\mathrm{E}$, is stable, there is a region D whose boundary includes the two stable manifolds of $\mathrm{E}$. such that any positive orbit inside $D$ tends to $E$, as $t$ tends to infinity and any positive orbit outside $\mathrm{D}$ intersects the positive $\mathrm{S}$-axis in finite time.

\section{CONCLUSION}

A Hopf bifurcation occurs when a periodic solution or limit cycle, surrounding an equilibrium point, arises or goes away as a parameter varies. When a stable limit cycle surrounds an unstable equilibrium point, the bifurcation is called a supercritical Hopf bifurcation. If the limit cycle is unstable and surrounds a stable equilibrium point then the bifurcation is called a subcritical Hopf bifurcation.

\section{REFERENCES}

Beretta, E. and Y. Takeuchi, 1995. Global stability of an SIR epidemic model with time delays. J. Math. Biol., s33: $250-260$.

Beretta, E. and Y. Takeuchi, 1997. Convergence results in SIR epidemic models with varying population sizes. Nonlinear Anal., 28: 1909-1921.

Beretta, E., T. Hara, W. Ma and Y. Takeuchi, 2001. Global asymptotic stability of an SIR epidemic model with distributed time delay. Nonlinear Anal. Theor. Meth. Appl., 47: 4107-4115.

Bogdanov, R., 1981a. Bifurcations of a limit cycle for a family of vector fields on the plan. Selecta Math. Soviet., 1: 373-388.

Bogdanov, R., 1981b. Versal deformations of a singular point on the plan in the case of zero Eigen-values. Selecta Math. Soviet., 1: 389-421.

Brauer, F. and P. van den Driessche, 2001. Models for transmission of disease with immigration of infectives. Math. Biosci., 171: 143-154.

Capasso, V. and G. Serio, 1978. A generalization of the Kermack-McKendrick deterministic epidemic model. Math. Biosci., 42: 43-61.

Derrick, W.R. and P. Van Den Driessche, 1993. A disease transmission model in a nonconstant population. J. Math. Bio., 31: 495-512.

Feng, Z. and H.R. Thieme, 1995. Recurrent outbreaks of childhood diseases revisited: The impact of isolation. Math. Biosci., 128: 93-130.

Hethcote, H.W. and D.W. Tudor, 1980. Integral equation models for endemic infectious diseases. J. Math. Bio., 9: $37-47$. 
Hethcote, H.W. and P. Van den Driessche, 1991. Some epidemiological models with nonlinear incidence. J. Math. Bio., 29: 271-287.

Hethcote, H.W. and S.A. Levin, 1989. Periodicity in Epidemiological Models. In: Applied Mathematical Biology, Levin, S.A., T.G. Hallam and L.J. Gross (Eds.). Springer, New York, USA., pp: 193-211.

Hethcote, H.W., H.W. Stech and P. Van Den Driessche, 1981. Nonlinear oscillations in epidemic models. SIAM. J. Appl. Math., 40: 1-9.

Hyman, J.M. and J. Li, 1998. Modeling the effectiveness of isolation strategies in preventing STD epidemics. SIAM. J. Appl. Math., 58: 912-925.

Kermack, W.O. and A.G. McKendrick, 1927. Contribution to the mathematical theory of epidemics, part I. Proc. R. Soc. London Ser. A, 115: 700-721.
Liu, W.M., H.W. Hethcote and S.A. Levin, 1987. Dynamical behavior of epidemiological models with nonlinear incidence rates. J. Math. Bio., 25: 359-380.

Ma, W., Y. Takeuchi, T. Hara and E. Beretta, 2002. Permanence of an SIR epidemic model with distributed time delays. Tohoku Math. J. Second Ser., 54: 581-591.

Ruan, S. and W. Wang, 2003. Dynamical behavior of an epidemic model with a nonlinear incidence rate. J. Differ. Equations, 188: 135-163.

Xiao, D. and S. Ruan, 2007. Global analysis of an epidemic model with nonmonotone incidence rate. Math. Biosci., 208: 419-429. 